\title{
Explicit but Not Implicit Mood is Affected by Progressive Social Exclusion
}

\author{
Erica J. Ho ${ }^{1,2}$, Gizem Surenkok, M. A. ${ }^{1}$, \& Vivian Zayas, Ph. D. ${ }^{1}$ \\ Cornell University ${ }^{1}$, Center for the Developing Brain, Child Mind Institute ${ }^{2}$
}

\begin{abstract}
Past research has shown that clear-cut exclusion (e.g., being completely left out) worsens mood and threatens basic needs, as assessed by explicit self-report measures. Here, we investigated the impact of subtle exclusion on implicit mood and self-esteem, as well as the effect of introspection on the same implicit processes. In five rounds of a virtual three-person ball-tossing game, all participants were initially included (round 1), but encountered increasing levels of either subtle exclusion or subtle overinclusion (rounds 2-5). Following each round, participants completed implicit measures; half of the participants were randomly assigned to also complete explicit measures before the implicit measures, which served to increase introspection. Replicating previous findings, progressive exclusion negatively impacted explicit moods and needs. However, neither exclusion nor introspection affected implicit mood and self-esteem. The construct validity of implicit measures is discussed.
\end{abstract}

Keywords: progressive social exclusion, ambiguous social dynamics, Cyberball, introspection, implicit mood

Des études ont démontré que l'exclusion catégorique (c.-à-d., être complètement mis à part) détériore l'humeur et menace les besoins de base, tel que mesuré par des instruments auto-rapportés. Cet article explore l'effet de l'exclusion subtile sur l'humeur et l'estime de soi implicites, ainsi que l'effet de compléter des mesures auto-rapportées sur ces processus implicites. Lors d'un jeu virtuel à trois joueurs comportant cinq parties, tous les participants étaient initialement inclus (partie 1) puis exposés à des niveaux croissants d'exclusion ou d'inclusion subtiles (partie 2-5). Après chaque partie, les participants ont complété des mesures implicites; la moitié des sujets ont été aléatoirement assignés à répondre à des mesures explicites afin d'accroitre leur sentiment d'introspection. Les résultats ont démontré qu'une exclusion progressive affecte négativement l'humeur et les besoins explicites. L'exclusion et l'introspection n'ont pas eu d'impact sur l'humeur implicite ou l'estime de soi. La validité de construit des mesures implicites sera discutée.

Mots-clés : exclusion sociale progressive, dynamique sociale ambigüe, Cyberball, introspection, humeur implicite

To be human is also to be interdependent. Human beings are social creatures who rely on each other to survive and thrive, making acceptance and belongingness absolutely vital to life. Not surprisingly, being rejected by others has been shown

This work was part of an honors thesis project completed by the first author, under the direction of the second and third authors. The authors would like to thank Drs. David Dunning and Corinna Löckenhoff for their feedback on the first author's thesis; Jason F. Anderson for providing the script for the progressive Cyberball paradigm; Dr. Jun Fukukura for methodological support; Joey Cheng, Frederico De Paoli, Corey Keane, Kyle Kurkela, Jasmine LaCoursiere, and Ming Xing for their assistance in data collection and entry; and Bryan Chong for technical support. We would also like to thank the Cornell Undergraduate Research Fund and the Einhorn Discovery Grant for funding this study. Please address correspondence to Erica Ho (email: eh366@cornell.edu) or Vivian Zayas (email: vz29@cornell.edu). to have a number of devastating mental and physical consequences (MacDonald \& Leary, 2005). However, to date, the vast majority of research on social exclusion has studied explicit responses to clear-cut exclusion - that is, what people consciously report feeling, as assessed via self-report methods, when they are completely and unambiguously excluded by others (for a review, see Williams, 2007). But in everyday scenarios, exclusion may be more ambiguous and subtle, such as when a person is only slightly left out of an interaction. One challenge for studying responses to subtle and ambiguous forms of exclusion is that using self-report methods, which directly ask individuals to report on their thoughts and feelings, might draw attention to dynamics that might have gone unnoticed otherwise. 
Thus, to investigate how an individual responds to subtle and ambiguous forms of social exclusion, the present study used implicit measures to assess mood and self-esteem. In contrast to explicit measures that directly ask participants to introspect, deliberate on, and report their feelings, implicit measures indirectly tap into how people feel and think. Our goals were twofold. First, we aimed to investigate whether subtle forms of social exclusion negatively impact implicit mood and self-esteem. That is, if people were not directly prompted to think about the dynamics, perhaps they would not be aware of these more subtle instances of exclusion, and would not experience a decrease in mood and self-esteem. Alternatively, because research has shown that people are highly sensitive to any signs of exclusion, perhaps they would experience a decrease in implicit mood and self -esteem no matter how subtle the dynamics. Second, we aimed to investigate whether prompting individuals to introspect, by having them complete self-report measures, draws attention to social dynamics, which in turn exacerbates the negative outcomes of exclusion on these same implicit measures. That is, does highlighting subtly negative dynamics (via self-report completion) lead people to feel even worse?

In the sections below, we first present a brief review of how past work has studied social exclusion. We then highlight questions, which past research has not addressed, that are the focus of the present work, and, afterwards, discuss our primary aims, hypotheses, and support for our expectations.

\section{The Effects of Clear-Cut Exclusion}

Traditionally, research on exclusion has focused on situations in which a person is clearly and unambiguously excluded. For example, in Cyberball (Williams, Cheung, \& Choi, 2000), which has become a classic paradigm for experimentally creating social exclusion, participants are told that they are to play a virtual turn-taking ball-tossing game with two other people. In fact, unbeknownst to the participants, the other two "players" do not actually exist, and their behaviors are programmed. In the standard exclusion condition, the other two players are programmed to throw the ball to the participant a few times at the very start of the game, but thereafter completely exclude the participant from the ball tossing and only throw the ball to each other.
Previous research using Cyberball and other methods (Baumeister, Twenge, \& Nuss, 2002) has found that being the target of clear-cut, unambiguous exclusion increases negative mood and lowers levels of basic fundamental needs such as feelings of belongingness, control, self-esteem, and meaningful existence (Williams, 2009). The experience also leaves one feeling pained (Eisenberger, Lieberman, \& Williams, 2003), cold (Zhong \& Leonardelli, 2008) and numb (DeWall \& Baumeister, 2006), and it increases the likelihood of engaging in antisocial behaviors (Twenge \& Campbell, 2003). Social exclusion has also been shown to increase cells' secretion of proinflammatory cytokines; in excess, these cytokines increase the body's susceptibility to inflammatory diseases (Slavich, Way, Eisenberger, \& Taylor, 2010) and have been implicated in the development of depressive symptoms (Anisman \& Merali, 2002). Lastly, unambiguous exclusion also activates areas of the brain (e.g., dorsal anterior cingulate cortex) similar to those activated during physical pain (Eisenberger et al., 2003).

\section{From Explicit Measures and Clear-Cut Exclusion to Implicit Measures and Subtle Exclusion}

Despite the rich body of research on the detrimental consequences of exclusion, past work, with few exceptions, has focused on instances of clear -cut exclusion (e.g., as created experimentally using Cyberball) and relied primarily on self-report measures to assess the consequences of exclusion on mood and self-esteem (Williams, 2009). But in day-today situations, exclusion may be much more subtle than in those situations involving clear-cut exclusion, and individuals are not typically asked to reflect on the experience and their feelings. Surprisingly, there has been much less attention devoted to understanding how people respond to and make sense of these subtler social dynamics, which, arguably, are likely to occur with regularity. For example, someone may be excluded by one person, but included by another one (Chernyak \& Zayas, 2010), or left out in only one part of an activity, but included in another part (Jones, Carter-Sowell, Kelly, \& Williams, 2009).

In the present research, we were interested in one form of subtle social dynamic: situations in which a person is included, but not to the same extent as the other people in the group, and excluded to a greater extent over time. To date, only two studies have examined responses to subtle forms of social exclusion. 


\section{EXCLUSION LOWERS EXPLICIT, NOT IMPLICIT MOOD}

This line of work used a modified version of Cyberball called the progressive Cyberball paradigm (Anderson \& Zayas, 2012; Löckenhoff, Cook, Anderson, \& Zayas, 2012). In this version, participants play five consecutive rounds of Cyberball. In the first round, participants are included by the two players and receive the ball one-third of the time. But, with each subsequent round, they receive fewer and fewer ball tosses until the fifth and final round, when they are nearly completely excluded.

Past research using this paradigm (Anderson \& Zayas, 2012; Löckenhoff et al., 2012) found that even small shifts from inclusion to exclusion (i.e., receiving four fewer ball tosses than a fair one-third of the tosses) had deleterious effects on mood and on psychological needs. However, critically, these studies used explicit measures in which participants were directly asked, following each round, to think about how they felt (e.g., belonging versus not belonging). But, asking individuals to report on their thoughts and feelings in response to subtle dynamics might draw their attention to various interpersonal undercurrents that they may otherwise have overlooked. Would participants have noticed receiving the ball four fewer times if they had not been prompted to think about whether they belonged (versus did not belong)?

To investigate how people respond to subtle instances of exclusion, it is necessary to use tools other than explicit measures. Implicit measures are one such tool, because they differ from self-reports in important ways. Self-report measures directly ask individuals to report on their feelings and thoughts in response to the social dynamics (e.g., "How much do you feel like you belong?"), and thus tap into what is referred to as explicit mood and self-esteem, which are defined respectively as consciously accessible mood and self-esteem that participants themselves report (Greenwald \& Banaji, 1995). In contrast, implicit measures assess individuals' states indirectly, with the aim of bypassing conscious deliberation and selfpresentational concerns. Thus, implicit measures are assumed to assess evaluations that become activated automatically, spontaneously, without effort, and, at times, without awareness (Bosson, Swann, \& Pennebaker, 2000; Quirin, Kazén, \& Kuhl, 2009).

Although self-reports of people's explicit, consciously-held beliefs, attitudes, and experiences are useful, their accuracy depends on people's ability to access their feelings and their willingness to report on them (Ross \& Nisbett, 1991). For this reason, while explicit and implicit measures at times show convergence, at other times they show dissociations (Greenwald, Poehlman, Uhlmann, \& Banaji, 2009). In particular, it has been argued that when topics are personally and socially sensitive, as might be the case for instances in which one experiences exclusion, people may misrepresent their feelings either to others for the sake of self-presentation or situational demands, or to themselves for the sake of defending against threat (Greenwald et al., 2009). The concern about situational demand characteristics is particularly relevant to the present work. As illustrated by Orne's (1962) "good subject effect," participants behave and respond in accordance with what they believe to be the true purpose of the experiment. Therefore, it is possible that in typical social exclusion studies, in which the social dynamic is obvious, participants may report more negative mood and lower self-esteem to conform to what they think the experimenter expects.

\section{How Do Varying Levels of Exclusion Affect Implicit Mood and Self-Esteem?}

In the present research, we were particularly interested in investigating whether experiencing varying levels of exclusion (from subtle to more extreme) would affect implicit self-esteem and implicit mood. In some instances, research has documented that people show an increase in implicit self-esteem in response to threats (Rudman, Dohn, \& Fairchild, 2007), but findings from the exclusion literature clearly predict that individuals will show a decrease in implicit mood and self-esteem. Specifically, according to the sociometer hypothesis (Leary, Tambor, Terdal, \& Downs, 1995), self-esteem is an automatic psychological meter for assessing whether one is included or excluded in a group. Although not using implicit measures, Leary and colleagues (1995) found that participants' ratings of their degree of inclusion in a social interaction were positively correlated with their self-esteem, and that their self-esteem decreased after experiencing social exclusion. In further support of our prediction, a different study, which focused specifically on implicit self-esteem, found that increasing participants' feelings of acceptance resulted in improvements in their implicit self-esteem (Baccus, Baldwin, \& Packer, 2004). Yet another study demonstrated that, after recalling a negative autobiographical memory, participants showed a significant decrease in implicit mood as assessed by the Implicit Positive and Negative Affect Test (IPANAT; Selcuk, Zayas, Günaydin, Hazan, \& Kross, 2012). 


\section{How Does Introspection Affect Implicit Mood and Self-Esteem in Response to Varying Levels of Exclusion?}

The present research also addressed a second related question: Does asking individuals to introspect on their feelings, for example by having them selfreport on their mood, in fact worsen their experience of exclusion as assessed by implicit mood and selfesteem? Although everyone may experience negative mood in situations in which the social exclusion is obvious, introspection may, in fact, worsen mood when the exclusion is subtle and ambiguous, because it may draw attention to only mildly negative affective states that may have been undetected otherwise.

The intuition that introspection may lead to worse outcomes in response to subtle forms of exclusion is supported by distinct research. In one study focusing specifically on the effects of introspection, participants were asked to pick between two types of posters that they would later take home (Wilson et al., 1993). Half of them were asked to think about the reasons for their selection, and the other half was not. When queried three weeks later, the group that had introspected was much less satisfied with their selection, compared to the control group. Similarly, participants who were told to introspect about the reasons for being in a current relationship were more likely to show changes in how they felt about their romantic partner, compared to those who did not introspect (Wilson \& Kraft, 1993). Research evaluating the efficacy of grief counseling also provides compelling evidence for the idea that introspection may, at times, be disadvantageous. Jordan and Neimeyer (2003) suggest in a meta-analysis of existing research that, in some cases, people may fare better without therapeutic intervention; in one study, men who participated in a group intervention for parents bereaved by the violent death of their children experienced worsened posttraumatic stress symptoms, compared to parents who did not receive the intervention (Murphy et al., 1998, as cited in Jordan \& Neimeyer, 2003). Collectively, the literature shows that introspection can destabilize people's attitudes, even about important and personally significant topics such as relationships with their significant others, or responses to death of a loved one.

\section{How Does Overinclusion Affect Implicit Mood and Self-Esteem?}

Finally, although not a central aim of the present work, we investigated a novel social dynamic of overinclusion, which was first identified by Anderson and Zayas (2012). In what they referred to as the overinclusion condition, participants were initially included by receiving the ball an equal number of times from the other players. Then, however, with each subsequent round, participants received more and more of the ball tosses, culminating in the final round in which they received nearly all the ball tosses. Interestingly, Anderson and Zayas (2012) found that overinclusion led to an overall increase in participants' mood and psychological need scores, but only in individuals characterized by high rejection sensitivity, who are chronically worried about rejection and belonging. In contrast, there was no appreciable effect of overinclusion for those with low rejection sensitivity.

\section{Aims and Hypotheses}

Whereas existing work on social exclusion has focused on how people respond to clear-cut exclusion as assessed by explicit measures of mood and psychological needs, the aim of the present work was to examine (i) whether exclusion would lead to more negative implicit mood and self-esteem, and (ii) whether answering self-report measures (versus not answering) worsens the experience of social exclusion. The present research also explored implicit responses to a novel social dynamic - overinclusion, in which one is included beyond the expected amount (i.e., receiving disproportionately more attention than the other members of one's social group; Anderson \& Zayas, 2012).

To address these aims, we manipulated subtle forms of exclusion (and overinclusion) by using the progressive Cyberball paradigm (Anderson \& Zayas, 2012; Löckenhoff et al., 2012). The use of this paradigm was necessary given the goal of investigating the effects of introspection on implicit moods and self-esteem; in particular, we reasoned that when exclusion is clear-cut, everyone is expected to respond with lowered moods and selfesteem. However, it is when exclusion is subtle and ambiguous - and open to interpretation - that being prompted to introspect may have an effect on people's implicit responses. Accordingly, although all participants experienced inclusion in the initial round, 


\section{EXCLUSION LOWERS EXPLICIT, NOT IMPLICIT MOOD}

they were randomly assigned to experience increasing levels of exclusion or overinclusion in successive rounds of the game. In addition, within both the exclusion and overinclusion conditions, participants were randomly assigned to either introspect - that is, to complete explicit self-report measures of their mood and needs following each round of the game or not to, prior to completing implicit mood and selfesteem measures ${ }^{1}$.

Based on the literature, we made several a priori predictions for responses to progressive exclusion. First, participants' levels of mood and psychological needs, both explicit and implicit, were expected to decline linearly with each round of Cyberball, as exclusion became more and more blatant. Second, extrapolating from the literature on the drawbacks of introspective thought, we predicted a greater linear decline in implicit mood and needs for participants who introspected (i.e., completed self-report measures) following each round, compared to those who did not.

Given that less is known about how individuals respond to dynamics involving overinclusion, and that the lone existing study on this topic found no overall effect of overinclusion, we did not make any a priori predictions and treated our analyses with regard to overinclusion as exploratory.

\section{Method}

\section{Participants}

One hundred and twenty-seven students at Cornell University participated in this experiment in exchange for either extra credit in a course or 5 USD prorated for every half-hour of participation. The data from one participant were discarded due to failure to properly follow directions. Data for the remaining 126 participants $\left(M_{\text {age }}=20.8, S D=2.0\right)$ are reported here; $71.7 \%$ are female; $57.9 \%$ White, $26.9 \%$ Asian, $11.9 \%$ Black/African American, and 3.1\% of other ethnicity.

\footnotetext{
${ }^{1}$ Past work has used explicit measures to assess mood and levels of psychological needs, one of which is self-esteem (the others are belonging, control, meaningful existence). The explicit measures used in our study were similar in this regard. In contrast, our implicit measures only assessed mood and one of the four needs, self -esteem. Thus, in this paper, explicit measures refers to measures assessing mood and psychological needs, whereas implicit measures refers to the measures assessing mood and self-esteem.
}

\section{Design}

This experiment employed a mixed design with two between-subject factors and one within-subject factor. The two overarching between-subject factors were self-report (present versus absent) crossed with inclusion status (progressive exclusion versus progressive overinclusion). The sample sizes within each cell ranged from 31 to 33 participants. In addition, the within-subject factor was departure from complete fair distribution of ball tosses. Specifically, as will be explained below, all participants played either five or six rounds and were fairly included in the first round. However, as will be also discussed in the procedures hereafter, participants experienced increasing levels of either exclusion or overinclusion in subsequent rounds depending on their randomly assigned condition.

\section{Procedure}

Upon their arrival in the laboratory, participants were told that their task was to take part in an online ball-tossing game (i.e., Cyberball; Williams et al., 2000) as part of a study on social interactions. The game was created using Inquisit 3.0.5.0 (Inquisit, 2011). Participants were informed that they would be playing with two other study participants seated elsewhere in the building. Unbeknownst to them was that the two other players did not actually exist, and their behaviors were, in fact, preprogrammed. Participants were then seated at a computer and asked to sign an informed consent form while the experimenter left the room for sixty seconds to check on the readiness of the other supposed experimenters and participants. Once the experimenter returned to the room, the game began. The upper corners of the game screen showed two silhouetted avatars of the cyber players labeled "Player A" and "Player C" ostensibly in order to protect their confidentiality; the bottom center of the screen showed an avatar labeled "You," which represented the participant. Participants used the computer mouse to click on the avatar of the player they wanted to throw the ball to. Participants were told that the computer task was one continuous game, which would be paused every few minutes to allow everyone to fill out questionnaires about the game experience. In actuality, each "pause" marked the end of a distinct round of Cyberball, each round comprising 54 ball tosses and lasting about 2.5 minutes. 
Participants were randomly assigned to either an explicit self-report condition or a no self-report condition. In the explicit self-report condition, participants completed questionnaires after each round of the game. These questionnaires consisted of explicit measures of mood and belonging needs answered on the computer, as well as implicit measures of mood and self-esteem answered on paper. In the no selfreport condition, participants completed only the implicit measures.

Within each of those conditions, participants were then randomly assigned to an inclusion status group: either exclusion or overinclusion. As in Anderson and Zayas' (2012) paradigm, the first round of the game for both groups consisted of fair inclusion, during which all three players received the ball one-third of the time, or 18 out of 54 tosses. In the exclusion group, participants received four (7.4\%) fewer tosses per round in each subsequent round (i.e., two fewer tosses from each player), such that participants received only two $(3.7 \%)$ of the total tosses in the fifth round. In the overinclusion group, participants received two $(3.7 \%)$ more tosses per round (i.e., one more toss from each of the players), such that participants received 26 out of 54 tosses $(48 \%)$ in the fifth round. Anderson and Zayas' (2012) rationale for the different ball toss increments between groups was to ensure that the study encompassed a full range of exclusion and overinclusion; receiving $3.7 \%$ of all tosses is near-total exclusion, whereas receiving $48 \%$ of all tosses is near-total overinclusion. At the end of the experiment, participants completed a manipulation check, a suspicion probe, and a demographics form. They were then debriefed on the purpose of the study and thanked for their time.

\section{Manipulation Checks and Suspicion Probe}

By the end of the game, participants were either being completely excluded or completely overincluded, depending on their condition. To assess their awareness of the imbalanced social dynamics, participants were asked to estimate the percentage of ball tosses they received from Player $\mathrm{C}$ and from Player A, and these estimates were averaged. We then investigated whether participants in the exclusion condition reported that the other two players had thrown the ball more often to one another, which would lead to lower estimations of having received the ball, and whether players in the overinclusion condition had thrown the ball more often to the participant, which would lead to higher estimations of having received the ball.

Participants were also asked the following openended question: "Do you think you have an idea of what this experiment is about?" They were then probed for previous knowledge regarding Cyberball and asked for their guesses on how Cyberball might have been used in the present experiment. No participant was able to guess the hypothesis of the experiment, but 17 participants (14 exclusion and 3 overinclusion; from these 17,8 were in the explicit self-report condition and 9 in the no self-report condition) reported suspicion that the implicit mood measures were being used to gauge their responses to being excluded or included in the game. The analyses were rerun excluding the participants who mentioned anything about one or more of the implicit mood measures being used to assess their mood following Cyberball, and the results remained relatively unchanged from those based on the entire sample.

\section{Controlling for Time-Related Effects}

Half of the participants played five rounds of the Cyberball game, as in Anderson and Zayas (2012). The other half, however, played six rounds, starting off with two rounds of fair play, or inclusion, rather than one, before the progressive exclusion or overinclusion rounds began. The reason for this design was to try to tease apart the confounded effects of time and exclusion. Participants' mood levels were expected to decline from progressive exclusion, but they may also have declined simply as a function of extended exposure to a tedious computer game. Thus, this staggered design, wherein some participants experienced the exclusion (or overinclusion) manipulation one round later than others, allowed the comparison of mood levels across manipulations, controlling for time. No effect of time was found. Therefore, all results were collapsed across stagger conditions.

\section{Measures}

Explicit measure of mood and psychological needs. After each round of Cyberball, participants in the explicit self-report condition completed the explicit mood and psychological needs scale, comprised of 12 randomly presented bipolar questions on a 7-point scale, also on the computer, following procedures used by Anderson and Zayas (2012). 


\section{EXCLUSION LOWERS EXPLICIT, NOT IMPLICIT MOOD}

The questions assessed level of needs for belonging ("disconnected/connected," "I belong/I don't belong," "like an outsider/like an insider"), control ("powerless/powerful," "I have control/I lack control," "uninfluential/influential"), mood ("sad/happy," "friendly/unfriendly," "angry/pleasant"), and awkwardness ("uneasy/easy," "comfortable/ uncomfortable," "awkward/not awkward"). In any given round of the game, Cronbach's alpha was greater than .72 for each subscale (i.e., belonging, control, mood, awkwardness) as well as for all 12 items. For the purpose of simplifying the results, a single aggregate mood-needs score, hereafter referred to as "explicit mood and needs," was used to represent the 12 items for each round (analyses examining the four individual scales produced similar results).

As in Anderson and Zayas (2012), the mood, belonging, and control items were based on Zadro, Williams, and Richardson's (2004) ostracism measure. In addition to belonging and control, self-esteem and meaningful existence are often implicated in social exclusion as well, but Anderson and Zayas (2012) excluded them for the sake of brevity; furthermore, a meta-analysis of rejection by Gerber and Wheeler (2009) suggested that out of these four needs, belonging and control were the most impacted by exclusion. Additionally, we used the awkwardness scale because it is appropriate for assessing negative feelings that might arise due to being overincluded; we included it in the exclusion condition for consistency across conditions.

Implicit measures of mood and self-esteem. Participants completed implicit measures following each round of Cyberball. Although there are a number of different types of implicit measures (Bosson et al., 2000), we used the following three, which have been used in past work to assess state changes in implicit mood and self-esteem: the Name Letter Task (Fukukura, Zayas, \& Ferguson, 2013; Kitayama \& Karasawa, 1997; Koole, Dijksterhuis, \& van Knippenberg, 2001), the IPANAT (Quirin et al., 2009; Selcuk et al., 2012), and the Signature Size Task (Zweigenhaft, 1977; Fukukura et al., 2013). Because we were interested in obtaining Signature Size Task as one of our implicit measures, participants completed all implicit measures using paper-and-pencil (versus computer). Those in the self-report condition completed the implicit measures following completion of the computer questionnaire, and those in the no self-report condition did so immediately following the Cyberball round.
Name Letter Task. Participants were given a list of the 26 letters of the Roman alphabet, in a different randomized order each time, and they were asked to rate their liking of each of them on a 7-point scale. To obtain a Name Letter Task score for each round, the mean of each participant's ratings of his or her firstname initial and surname initial was calculated, along with the mean ratings of the other 24 letters. The other -letters mean was then subtracted from the own-letters mean. Higher numbers reflect greater implicit positivity towards oneself (versus others).

IPANAT. Participants were shown words from a supposed artificial language (i.e., SAFME, VIKES, TUNBA, TALEP, BELNI, and SUKOV) and asked to indicate how well they thought it fit with six different adjectives: happy, helpless, energetic, tense, cheerful, and inhibited. Because of its length, the whole measure could not be shown after each round. Instead, participants rated one artificial word after each round. The specific artificial word presented in any given round was randomly selected, with no replacement. Within each round, Cronbach's alphas were greater than .78 for the three positive-IPANAT items and .72 for the three negative-IPANAT items. The correlations between the positive-IPANAT items and the negativeIPANAT items for any given round ranged from -.27 to .42 in the exclusion condition (10 out of the 25 correlations were significant at the $p=.05$ level, with $p$ values for the remaining correlations ranging from .107 to .996 , degrees of freedom ranged from 59 to 63 ), and from -.28 to .45 in the overinclusion condition ( 9 out of 25 correlations were significant at the $p=.05$ level, with $p$ values for the remaining correlations ranging from .055 to .926 , degrees of freedom ranged from 57 to 59). In addition, the results of analyses conducted separately for the positiveIPANAT and negative-IPANAT were highly similar. Thus, for the sake of simplicity and ease of presentation for each round, we computed one composite IPANAT score by reverse scoring the negative-IPANAT scores and averaging them with the positive-IPANAT scores.

Signature Size Task. The last measure in each set of questionnaires was always the Signature Size Task: at the very end of each round's questionnaires, we asked participants to sign their name on a line, ostensibly, to affirm that they had completed the preceding tasks to the best of their ability. To compute a signature score for each round, we first drew a rectangle around each signature, fully encompassing it such that the length captured the full horizontal 
breadth of the signature, and the width captured the full vertical stretch. We multiplied length and width together to calculate the area of the rectangle, which served as the Signature Size Task score.

Participants were excluded from the analysis of an implicit mood measure if their score for any of the rounds was three standard deviations or more away from the mean, or if one or more rounds of that measure had been left blank. This resulted in the exclusion of 8 participants in the exclusion condition ( 3 in the explicit self-report and 5 in the no self-report condition) and 6 participants in the overinclusion condition (3 explicit self-report, 3 no self-report). The analyses reported below reflect the exclusion of these outliers, but the results were relatively unchanged from analyses that did include them.

\section{Data Analytic Strategy}

All analyses were conducted within the exclusion and overinclusion conditions separately. Unless otherwise stated, general linear models (GLMs) with the five levels of round (that is, the degree of exclusion or overinclusion) as the within-subjects factor and self-report condition (that is, whether participants were asked to introspect or not) as the between-subjects factor were used for all primary dependent variables (e.g., fundamental needs in the explicit self-report condition, and implicit measures). The Greenhouse-Geisser correction for sphericity was performed for all instances in which sphericity was violated. Moreover, given the a priori prediction of a linear effect of round, as well as a linear interaction between round and self-report, the linear and quadratic contrasts for both the effect of round and the interaction were examined; only statistically significant linear or quadratic contrasts are reported here. Finally, follow-up planned t-tests were performed in order to examine differences between individual rounds.

The Kolgorov-Smirnov test for normality showed that our data did not significantly deviate from normal distributions, with the exception of the first- and second-round IPANAT scores in both the exclusion and overinclusion conditions ( $D$ s ranged from .12 to $.13, p$ s ranged from .014 to .044) and the third-, fourth-, and fifth-round Signature Size Task scores (Ds ranged from .11 to .18 , ps ranged from .001 to .021). Given these departures from normality, we ran all the GLMs again, but this time on logtransformed dependent variables. Results of the analyses using the log-transformed data were highly similar to the results obtained using the untransformed data. Furthermore, as a second approach for dealing with the non-normal distributions, we performed all analyses using the nonparametric Friedman's two-way ANOVA by ranks test. In contrast to GLM, Friedman's test does not assume that the dependent measures are normally distributed. Again, the results using the nonparametric tests produced similar results to the results of the GLMs on the untransformed data.

\section{Results}

\section{Manipulation Check: Do People Perceive Being Excluded or Overincluded?}

As expected, and replicating past work (Anderson \& Zayas, 2012; Löckenhoff et al., 2012), in the exclusion condition, participants indicated that the

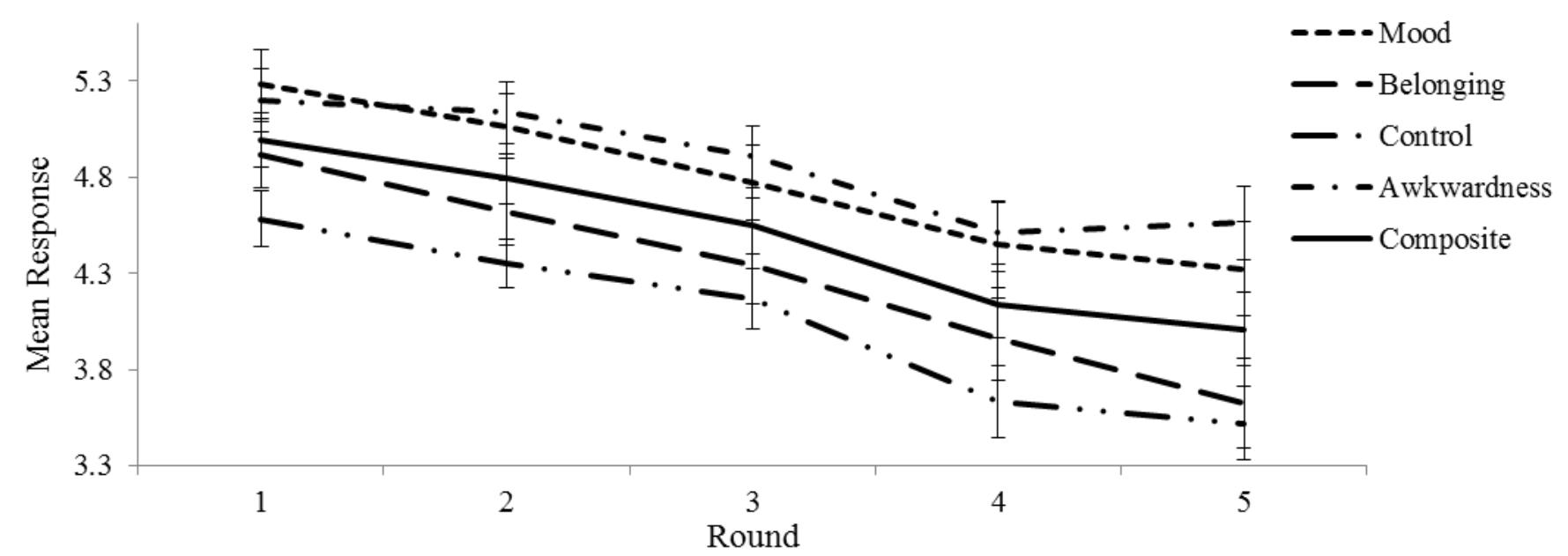

Figure 1. Changes in aggregate explicit mood and needs scores over the five rounds of progressive exclusion. In round 1, participants received $33.3 \%$ of the ball tosses, followed by $25.93 \%$ in round $2,18.52 \%$ in round $3,11.1 \%$ in round 4 , and $3.7 \%$ in round 5. Error bars indicate $\pm 1 S E$. 


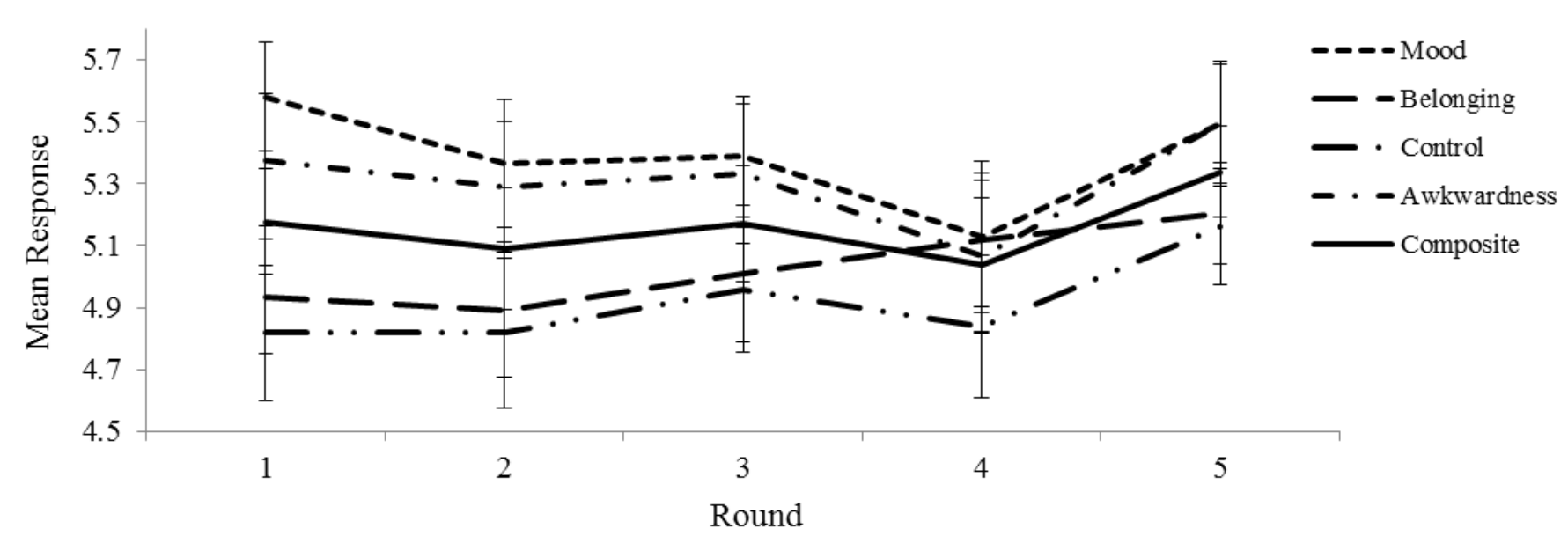

Figure 2. Changes in aggregate explicit mood and needs scores over the five rounds of progressive overinclusion. In round 1, participants received $33.3 \%$ of the ball tosses, followed by $37.04 \%$ in round $2,40.74 \%$ in round $3,44.44 \%$ in round 4 , and $48.15 \%$ in round 5. Error bars indicate $\pm 1 S E$.

other players had excluded them. They reported that they received the ball $25.64 \%(S D=12.16)$ of the time from the other two players, which is significantly less than $50 \%, t(63)=-16.03, p<.001$ (i.e., if participants had been included by both players, they would have reported receiving the ball from each player an average of $50 \%$ of the time). In the overinclusion condition, participants indicated that the other ostensible players had overincluded them. Participants reported that they received the ball an average of $57.05 \%(S D=11.44)$ of the time from the other two players, which is significantly greater than $50 \%, t(61)$ $=4.85, p<.001$.

\section{Replicating Past Work: Does Progressive Exclusion or Overinclusion Impact Explicit Mood and Needs?}

Before investigating the main questions of the present work, we aimed to assess the effects of progressive exclusion and progressive overinclusion on explicit mood and needs. This step is important because it provides evidence of the validity of the methods for manipulating social dynamics used in the present research.

As mentioned earlier, to simplify results, the explicit measures of mood, awkwardness, belonging,

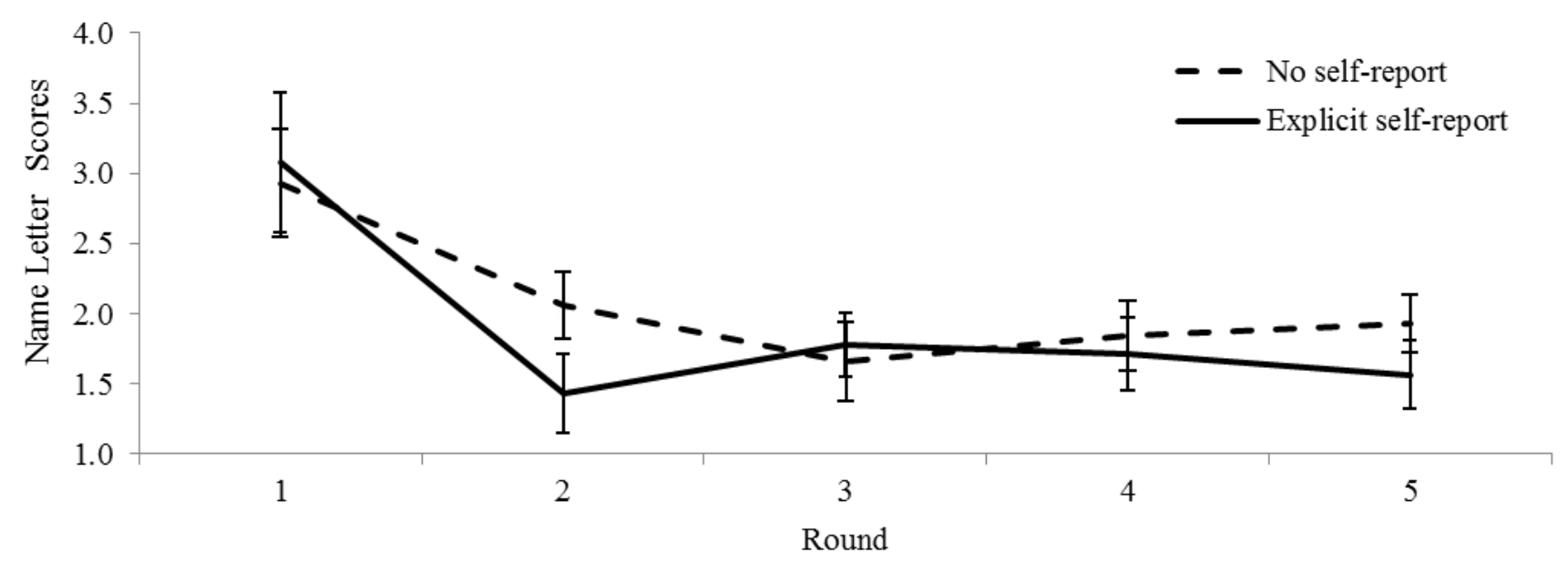

Figure 3. Changes in Name Letter scores over the five rounds of incremental exclusion. Error bars indicate $\pm 1 S E$. 


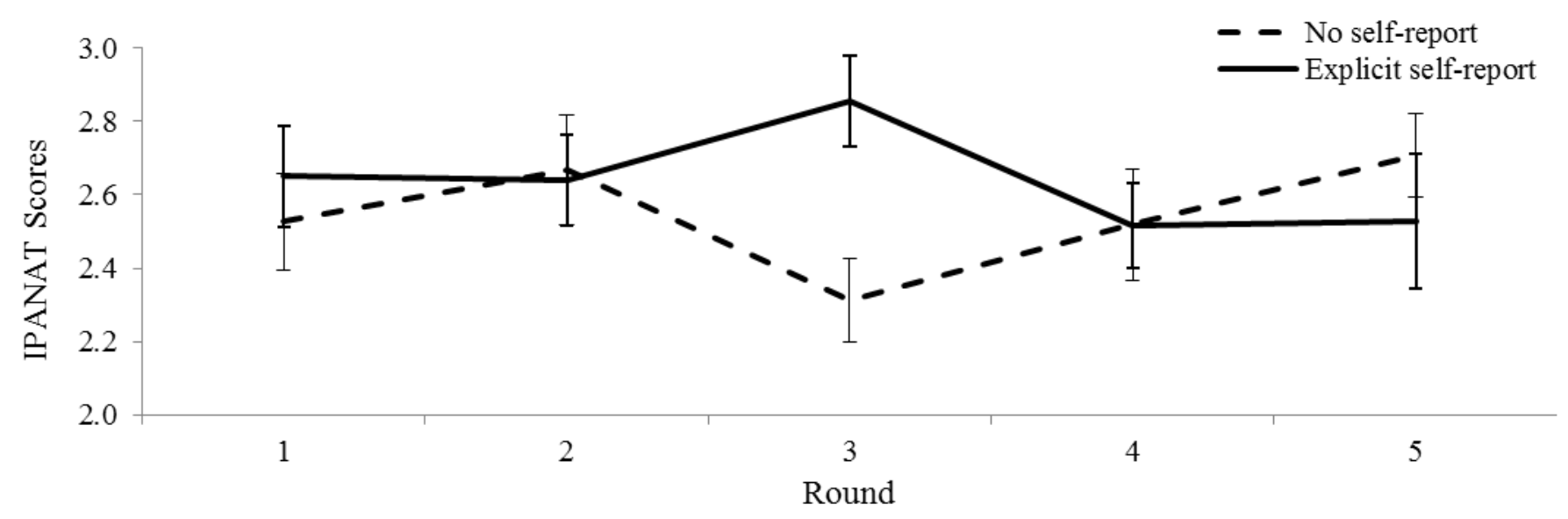

Figure 4. Changes in IPANAT scores over the five rounds of incremental exclusion. Error bars indicate $\pm 1 S E$.

and control were represented in our analyses with a single aggregate mood-needs score, hereafter referred to as "explicit mood and needs." In line with past work (Anderson \& Zayas, 2012), progressive exclusion from rounds 1 to 5 resulted in reduced explicit mood and needs, $F(1.78,55.1)=25.28$, $p<.001, \eta_{\mathrm{p}}{ }^{2}=.45$ (see Figure 1). A test of withinsubjects contrast for exclusion showed a linear effect, $F(1,31)=34.92, p<.001, \eta_{\mathrm{p}}^{2}=.53$.

Also, replicating previous work failing to show any effect of being overincluded, in response to being progressively overincluded from rounds 1 to 5 , participants did not experience an appreciable change in mood and needs, $F(2.45,73.54)=1.21, p=.310$, $\eta_{\mathrm{p}}^{2}=.04$ (see Figure 2).

\section{Beyond Explicit Measures: Does Progressive Exclusion or Overinclusion Impact Implicit Mood and Self-Esteem?}

Our main question was whether progressive exclusion would affect implicit mood and self-esteem, and if so, whether the effects would be more pronounced in the self-report condition in which participants are asked to think about their internal states. We predicted that in the exclusion condition, progressive exclusion would result in a linear decline in implicit mood and self-esteem scores, and that this decline may be more pronounced in the self-report condition as reflected by an interaction between magnitude of exclusion and self-report condition. In the next section, for ease of presentation and interpretation, we report results of analysis for the exclusion and overinclusion conditions separately. We discuss and interpret the meaning of these findings in the General Discussion section.

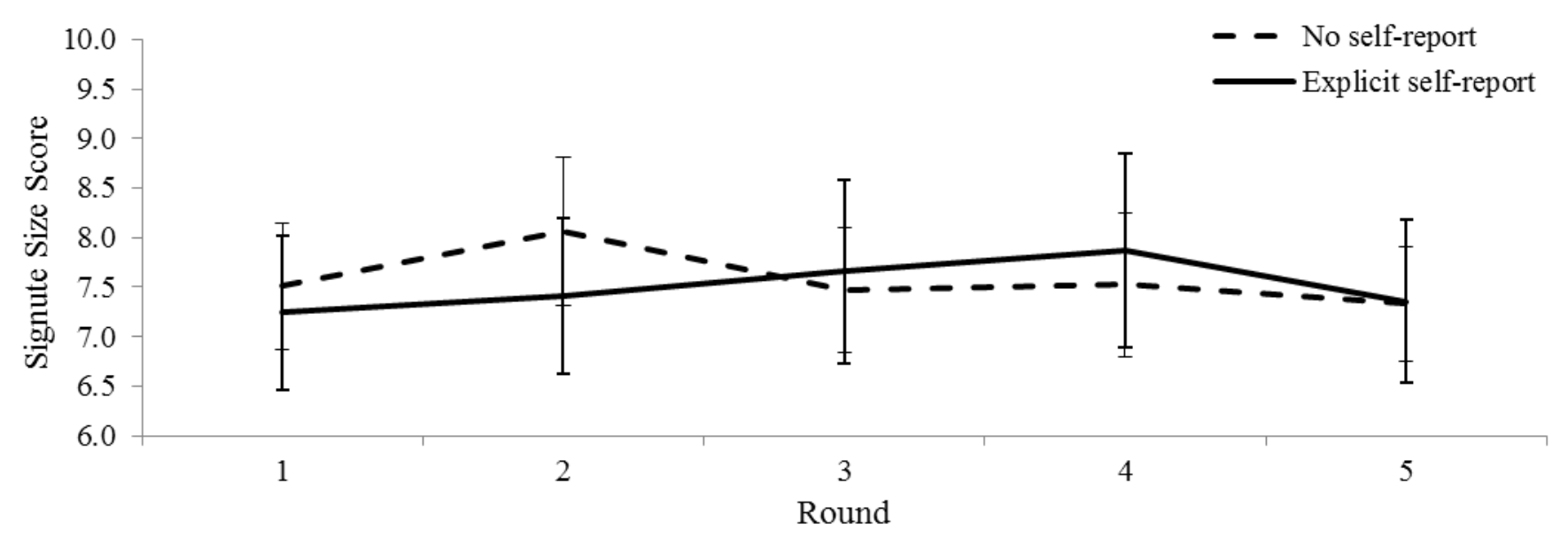

Figure 5. Changes in Signature Size scores over the five rounds of incremental exclusion. Error bars indicate $\pm 1 S E$. 


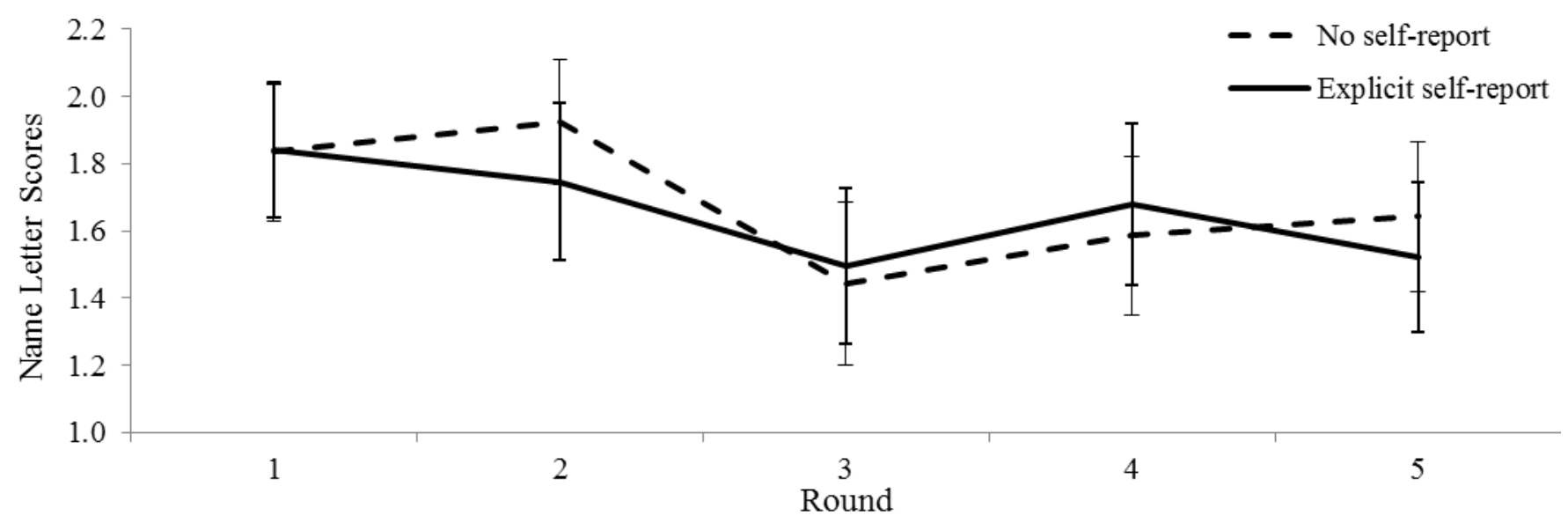

Figure 6. Changes in Name Letter scores over the five rounds of incremental overinclusion. Error bars indicate $\pm 1 S E$.

Does Progressive Exclusion Impact Implicit Mood and Self-esteem, and Is It Affected by Self-Report Condition?

Name Letter Task. Progressive exclusion from rounds 1 to 5 resulted in lower Name Letter Task scores, $F(1.48,90.18)=13.53, p<.001, \eta_{\mathrm{p}}^{2}=.18$, but the interaction between round and self-report condition was not significant, $F(1.48,90.18)=1.17$, $p=.304, \eta_{\mathrm{p}}{ }^{2}=.02$ (see Figure 3). Follow-up tests examining differences between the self-report conditions at each round of Cyberball showed no statistically significant differences (Cohen's $d$ ranged from .06 to .44; all $p \mathrm{~s}>.089$ ).

IPANAT. Progressive exclusion from rounds 1 to 5 did not result in lower IPANAT scores, $F(4,240)=$
$0.25, p=.907, \eta_{\mathrm{p}}{ }^{2}=.0004$; the interaction between round and self-report condition was also not significant, $F(4,240)=1.91, p=.109, \eta_{\mathrm{p}}{ }^{2}=.03$ (see Figure 4). Follow-up tests examining differences between the self-report conditions at each round of Cyberball showed a statistically significant difference in IPANAT scores at round $2, t(60)=3.25, d=.89$, $p=.002\left(M_{\text {explicit self-report }}=2.86, M_{\text {no self-report }}=2.31\right)$, which runs counter to predictions, and no statistically significant difference between groups for the other rounds (Cohen's $d$ ranged from .002 to .17; all $p \mathrm{~s}>$.399).

Signature Size Task. Progressive exclusion from rounds 1 to 5 did not result in smaller signatures, $F(3.06,171.13)=0.68, p=.569, \eta_{\mathrm{p}}{ }^{2}=.01$; the

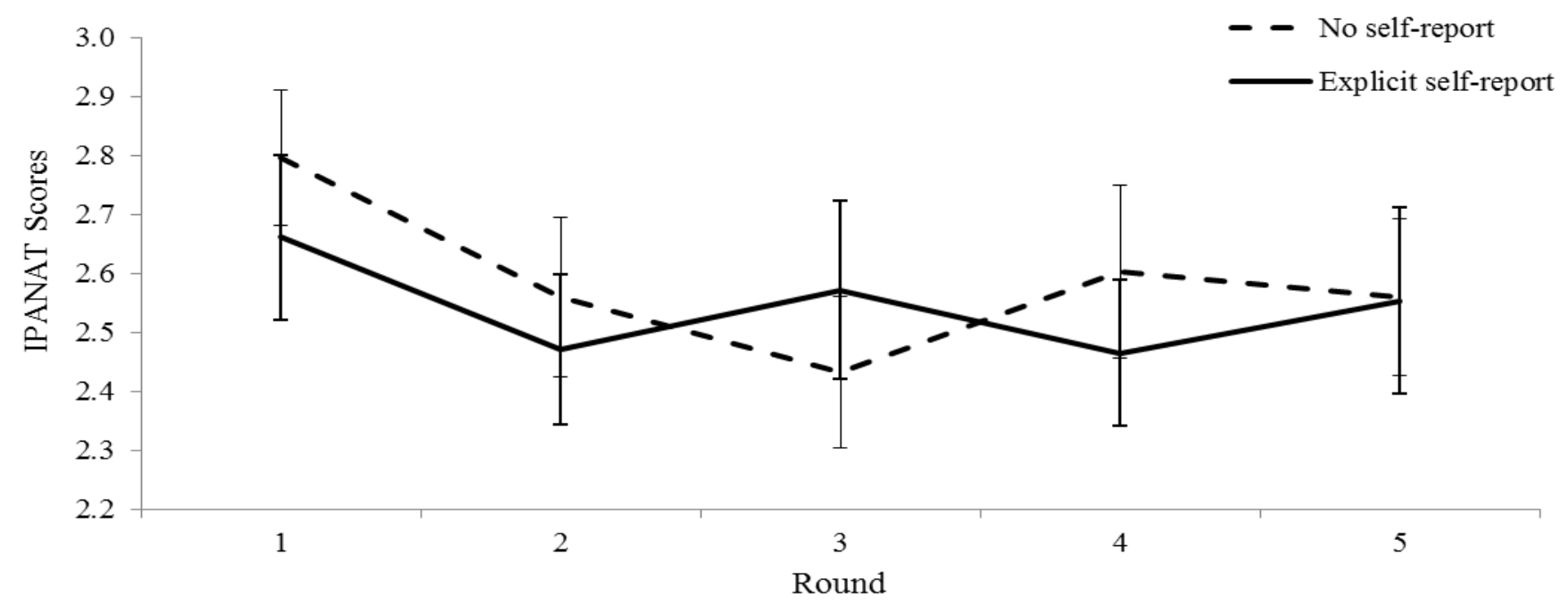

Figure 7. Changes in IPANAT scores over the five rounds of incremental overinclusion. Error bars indicate $\pm 1 S E$. 
interaction between round and self-report condition was also not significant, $F(3.06,171.13)=0.81$, $p=.492, \eta_{\mathrm{p}}{ }^{2}=.01$ (see Figure 5). Follow-up tests examining differences between the self-report conditions at each round of Cyberball showed no statistically significant differences (Cohen's $d$ ranged from .01 to .16; all $p \mathrm{~s}>.553$ ).

\section{Does Progressive Overinclusion Impact Implicit Mood and Self-Esteem, and Is It Affected by Self-Report Condition?}

Name Letter Task. Interestingly, progressive overinclusion from rounds 1 to 5 also resulted in lower Name Letter Task scores, $F(3.46,203.85)=2.72$, $p=.038, \eta_{\mathrm{p}}{ }^{2}=.04$, but the interaction between round and self-report condition was not significant, $F(3.46,203.85)=0.34, p=.824, \eta_{\mathrm{p}}{ }^{2}=.01$ (see Figure 6). A test of within-subjects contrasts for overinclusion showed a linear effect of round, $F(1,59)$ $=4.46, p=.039, \eta_{\mathrm{p}}^{2}=.07$, but no quadratic effect, $F(1,59)=1.58, p=.214, \eta_{\mathrm{p}}{ }^{2}=.03$. Follow-up tests examining differences between the self-report conditions of Cyberball showed no statistically significant differences (Cohen's $d$ ranged from .001 to .15 ; all $p \mathrm{~s}>.556$ ).

IPANAT. Progressive overinclusion from rounds 1 to 5 did not affect IPANAT scores, $F(4,228)=0.89$, $p=.474, \eta_{\mathrm{p}}{ }^{2}=.02$; the interaction between round and self-report condition was also not significant, $F(4,228)=0.35, p=.844, \eta_{\mathrm{p}}{ }^{2}=.01$ (see Figure 7$)$. Follow-up tests examining differences between the self-report conditions at each round of Cyberball showed no statistically significant differences (Cohen's $d$ ranged from .01 to .20 ; all $p \mathrm{~s}>.671$ ).
Signature Size Task. Progressive overinclusion from rounds 1 to 5 did not affect signature size, $F(3.52,193.43)=0.77, p=.530, \eta_{\mathrm{p}}{ }^{2}=.01$; the interaction between round and self-report condition was also not significant, $F(3.52,193.43)=0.79$, $p=.517, \eta_{\mathrm{p}}{ }^{2}=.01$ (see Figure 8). Follow-up tests examining differences between the self-report conditions at each round of Cyberball showed no statistically significant differences (Cohen's $d$ ranged from .0005 to .26 ; all $p$ s $>.345$ ).

\section{General Discussion}

To date, most research on social exclusion has examined explicit responses to exclusion, responses which participants deliberate about and report to the experimenter. As a result, the literature is well informed about people's conscious reactions to exclusion, but it knows relatively little about people's implicit reactions to exclusions, or about the impact of instructing people to reflect on exclusion. The primary aim of the present work was to examine changes in implicit mood and self-esteem as they unfold in a social dynamic involving ambiguous exclusion or overinclusion. The secondary aim was to explore the effect of introspection on those implicit processes, to examine whether drawing participants' attention to their social experience might enhance their processing of social cues.

To address these areas of inquiry, we adapted the procedures used by Anderson and Zayas (2012). Specifically, participants were either progressively excluded or overincluded in a modified version of the virtual ball-tossing game, Cyberball. Moreover, to assess participants' responses to ambiguous social

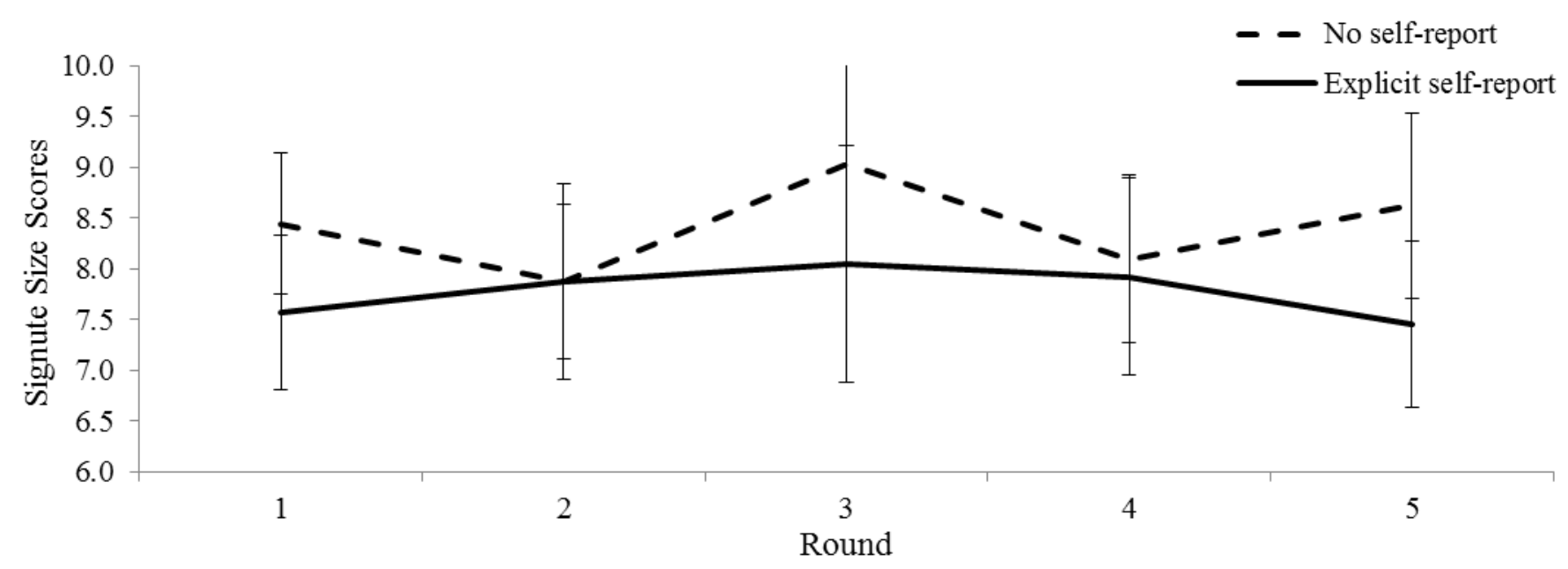

Figure 8. Changes in Signature Size scores over the five rounds of incremental overinclusion. Error bars indicate $\pm 1 S E$. 


\section{EXCLUSION LOWERS EXPLICIT, NOT IMPLICIT MOOD}

dynamics, we asked them to report on their feelings and moods resulting from being excluded and included; within each of the exclusion and overinclusion conditions, half of the participants completed both explicit and implicit measures of mood and needs following each round of the game, and the remaining half completed only implicit measures.

Based on Anderson and Zayas' (2012) work showing the impact of progressive social exclusion on explicit mood and needs, as well as based on the idea that explicit and implicit mood measures should tap into the same construct, we hypothesized that participants' implicit responses to exclusion and overinclusion should follow the same pattern as their explicit responses. We also predicted, based on the literature on the effects of introspection, that participants asked to self-report on their mood and psychological needs following each round of progressive exclusion would show worse implicit mood and self-esteem scores than participants not asked to self-report. Regarding the effects of selfreport following each round of progressive overinclusion, we made no a priori predictions, given that Anderson and Zayas (2012), the sole other study on this topic, found no overall effect of overinclusion on explicit mood; instead, we treated these analyses as exploratory. We did not find clear support for either of our hypotheses: first, neither progressive exclusion nor progressive overinclusion significantly affected participants' implicit measure scores. Contrary to our predictions, implicit mood and self-esteem did not clearly decline throughout progressive exclusion. Specifically, the IPANAT and Signature Size Task did not show declines with progressive exclusion. The only exception was the Name Letter Task, which did show decreases as a function of progressive exclusion. But, as we describe below, none of the implicit measures, even the Name Letter Task, showed evidence of construct validity. With regard to the overinclusion condition, implicit mood and self-esteem measures were relatively unaffected by progressive overinclusion. Here, too, only the Name Letter Task showed decreases as a function of progressive overinclusion, while the IPANAT and Signature Size Task did not. Second, there was no significant difference between implicit scores for participants who completed introspectioninducing self-reports versus no self-reports. Whereas we had expected introspection to exacerbate the effects of progressive exclusion, we instead found that there was no significant difference in implicit mood and self-esteem between the introspection groups, for both the exclusion and overinclusion conditions. In other words, completing self-reports following each round of the Cyberball game did not magnify (or dampen) responses on the implicit measures.

\section{Interpretation of The Null Results Involving Implicit Mood and Self-Esteem}

The finding that exclusion and overinclusion did not appreciably affect implicit mood and self-esteem is particularly surprising. One concern is that perhaps our methods differed from those used in previous work and this might have contributed to the null effect with regard to implicit mood. This possibility is highly unlikely given that the progressive exclusion and overinclusion manipulations did, in fact, work in the present study, successfully replicating past findings by Anderson and Zayas (2012) and Löckenhoff et al. (2012): participants' scores for mood and psychological needs in the explicit self-report condition were significantly affected by the exclusion manipulation, and not considerably altered by the overinclusion manipulation.

An alternative possibility might be a lack of statistical power to find the expected effect. But in both the exclusion and overinclusion conditions, the statistical power to detect a medium effect (Cohen's $d=.50$ ) was above .97 due to our relatively large sample sizes. Consequently, although null findings are not easily interpretable (i.e., it is not possible to claim "no effect"), we would have been very likely to detect it if exclusion or overinclusion had affected implicit mood and self-esteem to a moderate degree.

It is also possible that the implicit measures used in this study were not valid measures in this context. One way to assess a measure's construct validity is by examining how it correlates with other measures purportedly tapping into the same construct. In the present study, we would expect implicit measures to correlate with each other, given that they all reflect implicit mood and self-esteem. Additionally, we would also expect implicit measures to correlate with explicit measures of mood. Surprisingly, the present study found either weak or no correlations among the three implicit measures within a given round $(r s$ ranged from -.18 to .15; by comparison, $r s$ for the four subscales of the explicit measures ranged from .46 to .90). Moreover, the data showed either weak or no associations between the explicit and implicit measures ( $r s$ for round 1 scores for aggregated explicit 
mood and needs, and each of the implicit measures ranged from -.13 to .27), which clearly does not support the idea that the explicit and implicit measures tap into the same construct, at least to some extent. With 126 participants, our design allowed us to detect a medium-sized correlation $93.9 \%$ of the time; the fact that we did not find any significant correlation among the three implicit measures or between implicit and explicit measures suggests that the relationship between these theoretically related measures is, in actuality, trivial. These findings raise questions about the construct validity of these implicit measures and corroborate Bosson et al.'s (2000) work on the limited psychometric utility of some implicit self-esteem measures.

Moreover, given that there was an effect of exclusion on the Name Letter Task, it might be reasonable to assume that it is a more sensitive implicit measure compared to the IPANAT and Signature Size Task. However, a closer look at the data suggests that this is not the case, and that the effects observed on the Name Letter Task may be due less to effects of exclusion and more to effects of repeated administration. That is, the Name Letter Task did not correlate with the other two implicit measures in any of the rounds, and neither did it correlate with the explicit measures. This casts doubt on what the Name Letter Task is assessing. Moreover, not only was a linear trend over time observed in the exclusion condition, but a similar trend was also observed in the overinclusion condition. There is no reason that selfesteem should decrease as a result of being mildly more included. Thus, collectively, the data suggest that the finding involving the Name Letter Task is more likely to be related to the effect of time and repetition of the task than to the effect of being excluded (or overincluded) raise questions regarding the validity of such commonly used implicit measures. Refining existing and developing new implicit measures that assess mood, psychological needs, and self-esteem is an integral precursor to enhancing knowledge of how individuals perceive and respond to various social interactions, especially those that involve subtle social dynamics.

\section{Limitations and Future Directions}

Although the present work aimed to advance knowledge of responses to social dynamics in a number of ways - for example, by investigating responses to subtle exclusion using progressive exclusion, assessing implicit as well as explicit processes, and exploring responses to a novel social dynamic (overinclusion) - many questions remain for future work. A key finding in the present work was the absence of any effects of exclusion on implicit measures of mood and self-esteem. Future work should consider ways of potentially improving on the methodology. One possibility is to use measures with more reliable psychometric properties such as the Implicit Association Test (IAT: Greenwald, McGhee, \& Schwartz, 1998; Zayas \& Shoda, 2005). Another promising avenue for further elucidating the cognitive mechanisms underlying the response to progressive exclusion is to depend on more reliable unobtrusive measures. Recording continuous electroencephalogram (EEG) while participants complete the same tasks as in the present study may provide an ongoing, sensitive measure of the affective processes triggered by social exclusion. Critically, the use of EEG would not require directly asking participants about their feelings. A particular index of interest is frontal EEG asymmetry, which has been found to be associated with state-related emotional changes and responses (for a review, see Coan \& Allen, 2004). Specifically, greater right-versus-left frontal activation is associated with withdrawal behaviors and negative affect in general (Wheeler, Davidson, \& Tomarken, 1993). Future research might use right-versus-left frontal activation levels as an indicator of affective state.

\section{Conclusion}

Past research has consistently shown that human beings are extraordinarily in tune with signs of being included or excluded from their social groups, and can suffer both psychologically and physically when their belonging needs are threatened. However, most existing research has focused primarily on people's explicit responses to situations involving clear-cut, non-ambiguous social exclusion. The present study is one of the few studies to investigate the implicit processes that occur in response to situations involving more subtle and less obvious forms of exclusion, as well as to examine the possible impact of completing self-reports about mood and self-esteem on mood and self-esteem themselves.

The present study found that people are, indeed, very sensitive to subtle cues of exclusion, corroborating existing work (Anderson \& Zayas, 2012; Löckenhoff et al., 2012). Crucially, however, this sensitivity was only observed for explicit mood and psychological needs. Quite surprisingly, contrary 


\section{EXCLUSION LOWERS EXPLICIT, NOT IMPLICIT MOOD}

to expectation, implicit mood and self-esteem were relatively unaffected by subtle social exclusion and were not appreciably affected by introspection (i.e., completing self-reports). Given the high statistical power of our study to detect medium effects, and analyses of the psychometric properties of the implicit measures used in the present work, these null findings raise questions regarding the validity of such commonly used implicit measures. Refining existing and developing new implicit measures that assess mood, psychological needs, and self-esteem is an integral precursor to enhancing knowledge of how individuals perceive and respond to various social interactions, especially those that involve subtle social dynamics.

\section{References}

Anderson, J. F., \& Zayas, V. (2012). Even when subtle, exclusion costs all and overinclusion benefits some: Individuals high in rejection sensitivity experience psychological gains following overinclusion. Unpublished manuscript. Department of Psychology, Cornell University, Ithaca, New York, United States.

Anisman, H., \& Merali, Z. (2002). Cytokines, stress, and depressive illness. Brain, Behavior, and Immunity, 16, 513-524.

Baccus, J. R., Baldwin, M. W., \& Packer, D. J. (2004). Increasing implicit self-esteem through classical conditioning. Psychological Science, 15, 498-502.

Baumeister, R. F., Twenge, J. M., \& Nuss, C. K. (2002). Effects of social exclusion on cognitive processes: Anticipated aloneness reduces intelligent thought. Journal of Personality and Social Psychology, 83, 817-827.

Bosson, J. K., Swann, W. B., \& Pennebaker, J. W. (2000). Stalking the perfect measure of implicit self-esteem: The blind men and the elephant revisited? Journal of Personality and Social Psychology, 79, 631-643.

Chernyak, N., \& Zayas, V. (2010). Being excluded by one means being excluded by all: Perceiving exclusion from inclusive others during one-person social exclusion. Journal of Experimental Social Psychology, 46, 582-585.

Eisenberger, N. I., Lieberman, M. D., \& Williams, K. D. (2003). Does rejection hurt? An fMRI study of social exclusion. Science, 302, 290-292.
Fukukura, J., Zayas, V., \& Ferguson, M. J. (2013). The relationship between implicit and explicit materialism with self-esteem. Unpublished manuscript, Department of Psychology, Cornell University, Ithaca, New York: United States.

Gerber, J. P., \& Wheeler, L. (2009). On being rejected: A meta-analysis of experimental rejection research. Perspectives on Psychological Science, 4, 468-488.

Greenwald, A. G., \& Banaji, M. R. (1995). Implicit social cognition: Attitudes, self-esteem, and stereotypes. Psychological Review, 102, 4-27.

Greenwald, A. G., McGhee, D. E., \& Schwartz, J. L. K. (1998). Measuring individual differences in implicit cognition: The Implicit Association Test. Journal of Personality and Social Psychology, 74, 1464-1480.

Greenwald, A. G., Poehlman, T. A., Uhlmann, E. L., \& Banaji, M. R. (2009). Understanding and using the Implicit Association Test: III. Meta-analysis of predictive validity. Journal of Personality and Social Psychology, 97, 17-41.

Inquisit (Version 3.0.5.0) [Computer software]. Seattle, WA: Millisecond Software LLC.

Jones, E. E., Carter-Sowell, A. R., Kelly, J. R., \& Williams, K. D. (2009). 'I'm out of the loop': Ostracism through information exclusion. Group Processes and Intergroup Relations, 12, 157-174.

Jordan, J. R., \& Neimeyer, R. A. (2003). Does grief counseling work? Death Studies, 27, 765-786.

Kitayama, S., \& Karasawa, M. (1997). Implicit selfesteem in Japan: Name letters and birthday numbers. Personality and Social Psychology Bulletin, 23, 736-742.

Koole, S. L., Dijksterhuis, A., \& van Knippenberg, A. (2001). What's in a name? Implicit self-esteem and the automatic self. Journal of Personality and Social Psychology, 80, 669-685.

Leary, M. R., Tambor, E. S., Terdal, S. K., \& Downs, D. L. (1995). Self-esteem as an interpersonal monitor: The sociometer hypothesis. Journal of Personality and Social Psychology, 68, 518-530.

Löckenhoff, C. E., Cook, M. A., Anderson, J. F., \& Zayas, V. (2012). Age differences in responses to progressive social exclusion: The role of cognition and socioemotional functioning. Journals of Gerontology: Psychological Sciences, 68, 13-22.

MacDonald, G., \& Leary, M. R. (2005). Why does social exclusion hurt? The relationship between social and physical pain. Psychological Bulletin, 131, 202-223. 
Orne, M. T. (1962). On the social psychology of the psychological experiment: With particular reference to demand characteristics and their implications. American Psychologist, 17, 776-783.

Quirin, M., Kazén, M., \& Kuhl, J. (2009). When nonsense sounds happy or helpless: The Implicit Positive and Negative Affect Test (IPANAT). Journal of Personality and Social Psychology, 97, 500-516.

Ross, L., \& Nisbett, R. E. (1991). The person and the situation: Perspectives of social psychology. New York: McGraw-Hill Book Company.

Rudman, L. A., Dohn, M. C., \& Fairchild, K. (2007). Implicit self-esteem compensation: Automatic threat defense. Journal of Personality and Social Psychology, 93, 798-813.

Selcuk, E., Zayas, V., Günaydin, G., Hazan, C., \& Kross, E. (2012). Mental representations of attachment figures facilitate recovery following upsetting autobiographical memory recall. Journal of Personality and Social Psychology, 103, 362-378.

Slavich, G. M., Way, B. M., Eisenberger, N. I., \& Taylor, S. E. (2010). Neural sensitivity to social rejection is associated with inflammatory responses to social stress. Proceedings of the National Academy of Sciences, 107, 14817-14822.

Twenge, J. M., \& Campbell, W. K. (2003). “Isn't it fun to get the respect that we're going to deserve?', Narcissism, social rejection, and aggression. Personality and Social Psychology Bulletin, 29, 261272.

Wheeler, R. E., Davidson, R. J., \& Tomarken, A. J. (1993). Frontal brain asymmetry and emotional reactivity: A biological substrate of affective style. Psychophysiology, 30, 82-89.

Williams, K. D. (2007). Ostracism. Annual Review of Psychology, 58, 425-452.

Williams, K. D. (2009). Ostracism: A temporal needthreat model. In M. Zanna (Ed.), Advances in experimental social psychology (pp. 279-314). NY: Academic Press.

Williams, K. D., Cheung, C. K. T., \& Choi, W. (2000). Cyberostracism: Effects of being ignored over the Internet. Journal of Personality and Social Psychology, 79, 748-762.

Wilson, T. D., \& Kraft, D. (1993). Why do I love thee?: Effects of repeated introspections about a dating relationship on attitudes toward the relationship. Personality and Social Psychology Bulletin, 19, 409-418.
Wilson, T. D., Lisle, D. J., Schooler, J. W., Hodges, S. D., Klaaren, K. J., \& LaFleur, S. J. (1993). Introspecting about reasons can reduce post-choice satisfaction. Personality and Social Psychology Bulletin, 19, 331-331.

Zadro, L., Williams, K., \& Richardson, R. (2004). How low can you go? Ostracism by a computer is sufficient to lower self-reported levels of belonging, control, self-esteem, and meaningful existence. Journal of Experimental Social Psychology, 40, 560-567.

Zayas, V. \& Shoda, Y. (2005). Do automatic reactions elicited by thoughts of romantic partner, mother, and self relate to adult romantic attachment? Personality and Social Psychology Bulletin, 31, 10111025.

Zhong, C. B., \& Leonardelli, G. J. (2008). Cold and lonely: Does social exclusion literally feel cold? Psychological Science, 19, 838-842.

Zweigenhaft, R. L. (1977). The empirical study of signature size. Social Behavior \& Personality: An International Journal, 5, 177-185.

Received October 1, 2013

Revision received January 11, 2014

Accepted January 21, 2014 